Probing phonons in plutonium

M. Krisch, D. Farber, F. Occelli, A. Schwartz, T.C. Chiang, M. Wall, C. Boro, R. Xu

December 22, 2003

Symposium on Actinides: Science and Technology Boston, MA, United States December 1, 2003 through December 3, 2003 
This document was prepared as an account of work sponsored by an agency of the United States Government. Neither the United States Government nor the University of California nor any of their employees, makes any warranty, express or implied, or assumes any legal liability or responsibility for the accuracy, completeness, or usefulness of any information, apparatus, product, or process disclosed, or represents that its use would not infringe privately owned rights. Reference herein to any specific commercial product, process, or service by trade name, trademark, manufacturer, or otherwise, does not necessarily constitute or imply its endorsement, recommendation, or favoring by the United States Government or the University of California. The views and opinions of authors expressed herein do not necessarily state or reflect those of the United States Government or the University of California, and shall not be used for advertising or product endorsement purposes. 


\title{
Probing Phonons in Plutonium
}

\author{
Joe Wong ${ }^{1, *}$, Michael Krisch ${ }^{2}$, Daniel L. Farber ${ }^{1}$, Florent Occelli ${ }^{1}$, Adam J. Schwartz ${ }^{1}$, Tai \\ C. Chiang ${ }^{3}$, Mark Wall ${ }^{1}$, Carl Boro ${ }^{1}$, and Ruqing $\mathrm{Xu}^{3}$ \\ ${ }^{1}$ Lawrence Livermore National Laboratory, University of California, PO Box 808, \\ Livermore, CA 94551, USA \\ ${ }^{2}$ European Synchrotron Radiation Facility, BP 220, F-38043 Grenoble Cedex, France \\ ${ }^{3}$ Department of Physics and Frederick Seitz Materials Research Laboratory, \\ University of Illinois at Urbana-Champaign, , Urbana, IL 61801, USA
}

Plutonium $(\mathrm{Pu})$ is well known to have complex and unique physico-chemical properties [1]. Notably, the pure metal exhibits six solid-state phase transformations with large volume expansions and contractions along the way to the liquid state: $\alpha \rightarrow \beta \rightarrow \gamma \rightarrow \delta \rightarrow \delta^{\prime} \rightarrow \varepsilon \rightarrow$ liquid. Unalloyed $\mathrm{Pu}$ melts at a relatively low temperature $\sim 640^{\circ} \mathrm{C}$ to yield a higher density liquid than that of the solid from which it melts, (Figure 1). Detailed understanding of the properties of plutonium and plutonium-based alloys is critical for the safe handling, utilization, and long-term storage of these important, but highly toxic materials. However, both technical and and safety issues have made experimental observations extremely difficult.
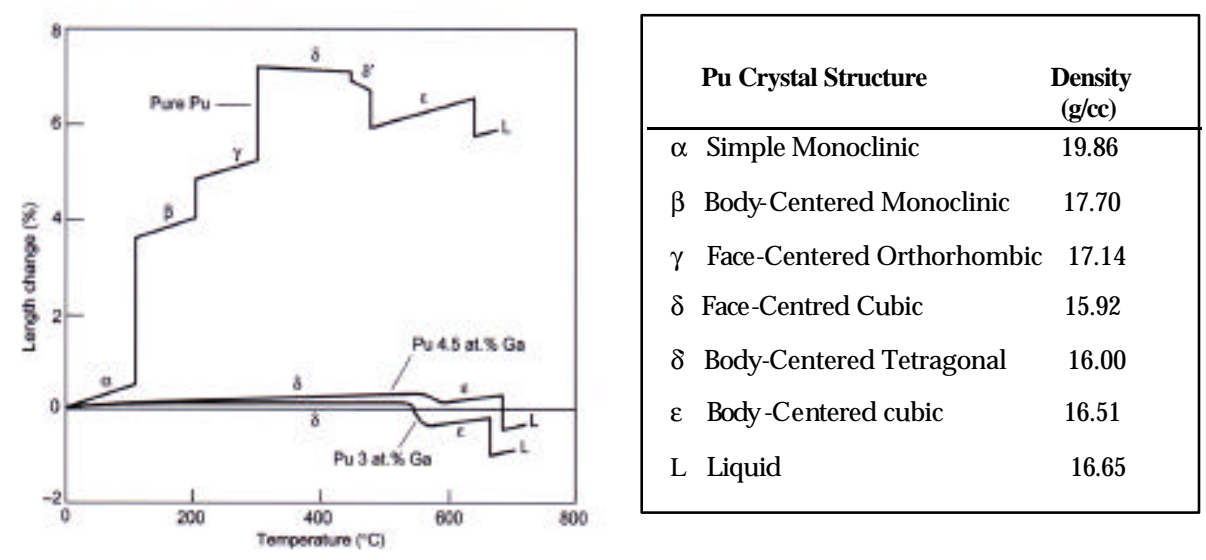

Figure 1 Transformations in $\mathrm{Pu}$ to different crystal structures are accompanied by very large volume changes. Alloying with $\mathrm{Ga}$ or $\mathrm{Al}$ avoids the transformation to $\gamma, \beta$ and $\alpha$ and stabilizes the $\delta$ phase all the way to room temperature and below [1]. 
Phonon dispersion curves (PDCs) are key experimental data to the understanding of the basic properties of $\mathrm{Pu}$ materials such as: force constants, sound velocities, elastic constants, thermodynamics, phase stability, electron-phonon coupling, structural relaxation, etc. However, phonon dispersion curves (PDCs) in plutonium $(\mathrm{Pu})$ and its alloys have defied measurement for the past few decades since the discovery of this element in 1941. This is due to a combination of the high thermal-neutron absorption cross section of plutonium and the inability to grow the large single crystals (with dimensions of a few millimeters) necessary for inelastic neutron scattering. Theoretical simulations of the Pu PDC continue to be hampered by the lack of suitable inter-atomic potentials. Thus, until recently the PDCs for Pu and its alloys have remained unknown experimentally and theoretically.

The experimental limitations have recently been overcome by using a tightly focused undulator x-ray micro-beam scattered from single-grain domains in polycrystalline specimens. This experimental approach has been applied successfully to map the complete PDCs of an fcc $\delta$-Pu-Ga alloy using the high resolution inelastic xray scattering (HRIXS) capability on ID28 [2].

The complete PDCs for an fcc Pu-0.6 wt\% Ga alloy are plotted in Figure 2, and represent the first full set of phonon dispersions ever determined for any $\mathrm{Pu}$-bearing materials [3]. The solid curves (red) are calculated using a standard Born-von Kármán (B-vK) force constant model. An adequate fit to the experimental data is obtained if interactions up to the fourth-nearest neighbours are included. The dashed curves (blue) are recent dynamical mean field theory (DMFT) results by Dai et al. [4].

The elastic moduli calculated from the slopes of the experimental phonon dispersion curves near the $\Gamma$ point are: $\mathrm{C}_{11}=35.3 \pm 1.4 \mathrm{GPa}, \mathrm{C}_{12}=25.5 \pm 1.5 \mathrm{GPa}$ and $\mathrm{C}_{44}=30.53 \pm 1.1$ GPa. These values are in excellent agreement with those of the only other measurement on a 


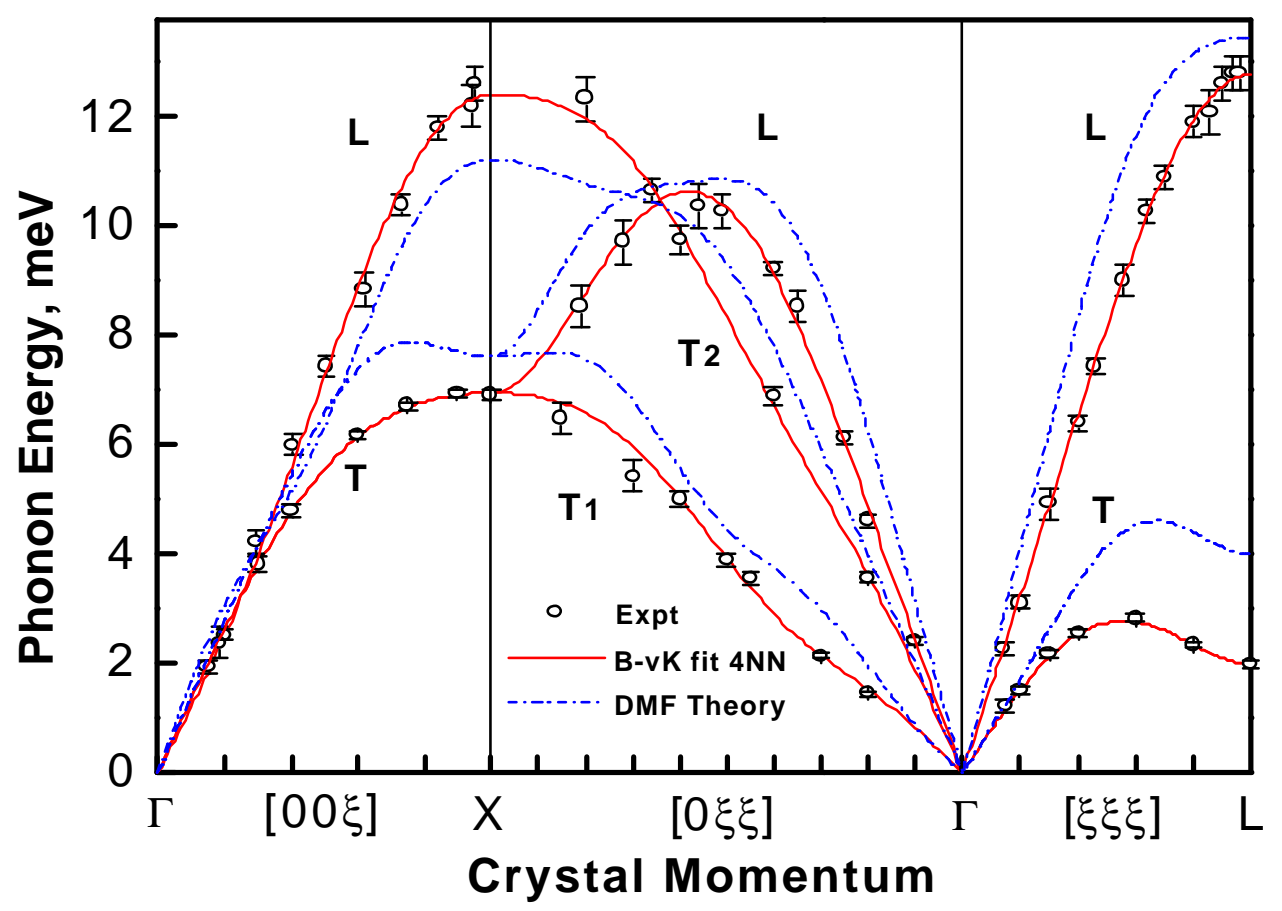

Fig. 2. Phonon dispersions along high symmetry directions in $\delta$-Pu- $0.6 \mathrm{wt} \% \mathrm{Ga}$ alloy. The longitudinal and transverse modes are denoted $\mathrm{L}$ and $\mathrm{T}$ respectively. The experimental data are shown as circles. Along the $[0 \xi \xi]$ direction, there are two transverse branches $[011]<01-1>\left(\mathrm{T}_{1}\right)$ and $[011]<100>\left(\mathrm{T}_{2}\right)$. Note the softening of the TA[ $\xi \xi \xi]$ branch towards the L point. The lattice parameter of our samples is a=0.4621 $\mathrm{nm}$. The red solid curves are the fourth nearest neighbour Born-von Kármán model fit. The blue dashed curves are calculated dispersions for pure $\delta$-Pu based on DMFT (4).

similar alloy (1 wt \% Ga) using ultrasonic techniques [5] as well as with those recently calculated from a combined DMFT and linear response theory for pure $\delta$-Pu [4]

Several unusual features, including a large elastic anisotropy, a small shear elastic modulus $C^{\prime}$, a Kohn-like anomaly in the $\mathrm{T}_{1}[011]$ branch, and a pronounced softening of the [111] transverse modes are found. These features can be related to the phase transitions of plutonium and to strong coupling between the lattice structure and the $5 f$ valence instabilities. The HRIXS results also provide a critical test for theoretical treatments of highly correlated 5f electron systems as exemplified by recent dynamical mean field theory (DMFT) calculations for $\delta$-plutonium. 
The experimental-theoretical agreements shown in Figure 2 in terms of a low shear elastic modulus C', a Kohn-like anomaly in the T1[011] branch, and a large softening of the T[111] modes give credence to the DMFT approach for the theoretical reatment of $5 f$ electron systems of which $\delta$-Pu is a classic example. However, quantitative differences remain. These are the position of the Kohn anomaly along the $T_{1}[011]$ branch, the energy maximum of the $T[111]$ mode s and the softening of the calculated $T[100]$ branch near the $X$ point, which is not observed experimentally. These differences are significant and thus provide a framework for refined theoretical treatments. Systematic HRIXS experiments as a function of temperature and concentration in the fcc $\mathrm{Pu}-\mathrm{Ga}$ alloys are underway.

This work was performed under the auspices of the U. S. Department of Energy by the University of California, Lawrence Livermore National Laboratory under Contract No. W-7405-Eng-48 and the U.S. Department of Energy by the University of Illinois Frederick Seitz Materials Research Laboratory under Grant No. DEFG02-91ER45439. We are thankful to Francesco Sette for his support and encouragement in this project, and to P. Berkvens and P. Colomp for their advice and technical assistance.

\section{References}

[1] S.S. Hecker, Challenges in Plutonium Science, Los Alamos Sci, 26, 290 (2000).

[2] H. Requardt, et al. Phys. Rev.B 66, 214303 (2002)

[3] Joe Wong et al. Science, 301, 1078 (2003)

[4] X. Dai et al., Science 300, 953 (2003)

[5] H. M. Ledbetter and R.L. Moment, Acta Metall. 24, 891 (1976)

\footnotetext{
* To whom correspondence should be addressed. E-mail wong10@llnl.gov
} 